\title{
Role of poly(styrene-acrylonitrile) copolymer interface layer in controlling charge storage and decay properties of amorphous selenium films
}

\author{
S. Chand, ${ }^{\text {a) }}$ G. D. Sharma, and S. Dwivedi \\ National Physical Laboratory, Dr. K. S. Krishnan Marg, New Delhi 110012, India
}

(Received 18 January 2006; accepted 21 February 2006; published online 24 May 2006)

\begin{abstract}
We report here the effect of poly(styrene-acrylonitrile) copolymer interface barrier layer films on the charge storage and decay characteristics of amorphous selenium $(a-\mathrm{Se})$ films studied by the thermally stimulated discharge current and potential decay techniques, respectively. Incorporation of copolymer interface barrier layer reduces the charge storage capacity/built-up of residual potential in $a$-Se film and enhances its x-ray sensitivity. These effects have been attributed to the blocking and field assisted enhanced mobility role of the copolymer interface barrier film. (c) 2006 American Institute of Physics. [DOI: 10.1063/1.2193045]
\end{abstract}

Analogue as well as digital selenium radiographies use $^{1-4}$ amorphous selenium $(a-\mathrm{Se})$ as an imaging material in active matrix/multilayer array forms. To make selenium technology more versatile, there is a need to develop better sensitive and cost effective imaging materials. International efforts are being $\operatorname{made}^{5-10}$ in this direction by way of modulating intrinsically the imaging properties of $a$-Se films such as enhancement in x-ray sensitivity $(S)$ by alloying, doping, etc. However, our recent efforts to make $a$-Se better sensitive are based on a new concept, i.e., incorporating an interface barrier layer of an insulating copolymeric material in combination with $a$-Se films. This letter reports, for the first time, the effect of a copolymer (rather than a single polymer) of styrene and acrylonitrile, i.e., poly(styreneacrylonitrile) copolymer $\mathrm{P}(\mathrm{S}: \mathrm{AN})$ on the charge storage and decay properties of $a$-Se films. In fact, considerable reduction in charge storage capacity/built-up of residual potential and subsequent enhancement in $S$ of $a$-Se films have been observed by incorporating vacuum deposited interface barrier layer of this copolymer. The charge storage and decay behavior of $a$-Se films have been studied in the temperature range of $295-385 \mathrm{~K}$ as a function of $\mathrm{P}(\mathrm{S}: \mathrm{AN})$ interface barrier layer having thickness in the range of 0-10 $000 \AA$ using thermally stimulated discharge current (TSD) technique. The reduction in charge storage capacity found in TSD studies has further been supplemented by direct measurements of built-up of residual potential in $a$-Se films as a function of $\mathrm{P}(\mathrm{S}: \mathrm{AN})$ film thickness ( 2500-10 $000 \AA)$. The mechanism for the observed reduction in charge storage capacity/built-up of residual potential and subsequent enhancement in $S$ of $a$-Se films has been discussed in this short Communication.

Copolymer $\mathrm{P}(\mathrm{S}: \mathrm{AN})$ having 75:25 ratio was obtained from Polysciences Inc., USA. Its thin films in the range of 2500-10000 $\AA$ were vacuum deposited onto ultrasonically cleaned aluminum $(\mathrm{Al})$ substrates of size of $50 \times 50 \times 1 \mathrm{~mm}^{3}$ at vacuum of $\sim 10^{-5}$ torr. The films so deposited were annealed at temperature of about $383 \mathrm{~K}$ for about $1 \mathrm{~h}$ to ensure better adherence with the substrate. Subsequently, on top of these $\mathrm{P}(\mathrm{S}: \mathrm{AN})$ films, thick films $(100 \mu \mathrm{m})$ of $a$-Se were also vacuum deposited under vacuum of $\sim 10^{-5}$ torr as described previously. ${ }^{11,12}$ Thus a sample configuration of $\mathrm{Al} / \mathrm{P}(\mathrm{S}: \mathrm{AN}) / a$-Se was obtained. It

\footnotetext{
${ }^{a)}$ Electronic mail: schand@mail.nplindia.ernet.in
}

may, however, be mentioned here that even after thermal annealing of $\mathrm{P}(\mathrm{S}: \mathrm{AN})$ films the adherence of $\mathrm{P}(\mathrm{S}: \mathrm{AN})-a$-Se combinational films with Al substrate deteriorated considerably for thickness greater than $10000 \AA$. In fact, beyond this thickness the samples showed pealing off tendency prior to the measurements, hence the investigations were restricted only to $10000 \AA$ films of $\mathrm{P}(\mathrm{S}: \mathrm{AN})$. The $\mathrm{Al} / \mathrm{P}(\mathrm{S}: \mathrm{AN}) / a$-Se samples so obtained were rested in dark for 3 months for stabilization of stray effects, if any and then used for TSD measurements. TSD measurements were made by putting a pressure electrode on top of a polarized sample and heating at the rate of $5 \mathrm{~K} / \mathrm{min}$. The current so released was measured by Keithley 610C Electrometer for plotting the TSD spectra. Next the potential decay measurement was made by corona charging these samples and measuring the potential in dark as well as on $\mathrm{x}$-ray exposure $[50 \mathrm{kV}$ (peak), $10 \mathrm{mAs}$ ] using a calibrated probe coupled with Keithley $610 \mathrm{C}$ Electrometer. The rate of exposure was measured using Keithley dosimeter and it was $\sim 26 \times 10^{-3} \mathrm{R} / \mathrm{s}$.

To study the TSD behavior of $\mathrm{Al} / \mathrm{P}(\mathrm{S}: \mathrm{AN}) / a$-Se films, these were polarized by a mechanism reported earlier ${ }^{13,14}$ and shown again in Fig. 1 for the sake of convenience of understanding the results and discussion of the present letter. In this mechanism the samples were first irradiated with white light from a $100 \mathrm{~W}$ tungsten filament for about 30 min. A water filter was used to cut off the IR radiation. The intensity of illumination was about 1000 1x. The irradiation produced reversible photostructural defects of type ${ }^{15-17}$ $\mathrm{C}_{3}{ }^{+}$and $\mathrm{C}_{1}{ }^{-}$on the surface of $a$-Se film up to a depth of a few microns. After the exposure the films were charged positively under corona discharge. This resulted in the built-up of

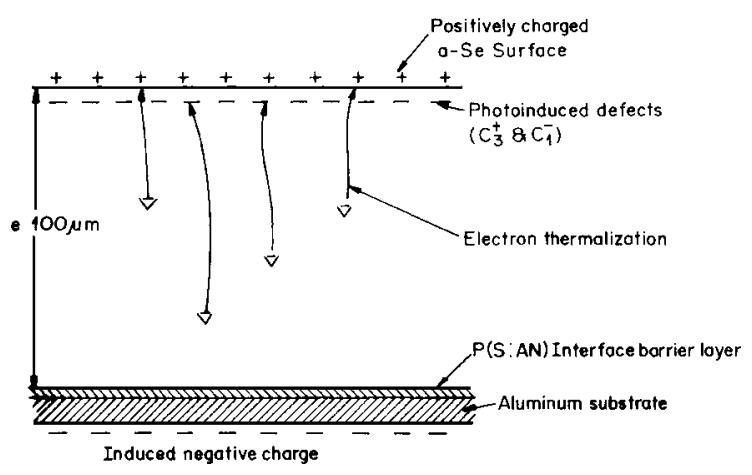

FIG. 1. Mechanism of polarization in $a$-Se films with an interface layer of $\mathrm{P}(\mathrm{S}: \mathrm{AN})$. 


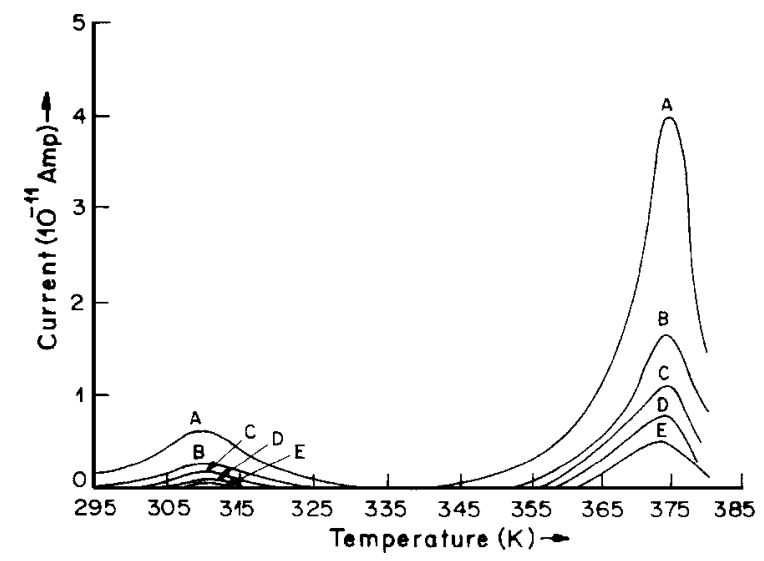

FIG. 2. TSD spectra of irradiated and positively charged $a$-Se films $(\sim 100 \mu \mathrm{m}$ thick) with and without $\mathrm{P}(\mathrm{S}: \mathrm{AN})$ interface layer. Curve A corresponds to films without an interface layer and curves B, C, D, and E correspond to films with interface layers of $\mathrm{P}(\mathrm{S}: \mathrm{AN})$ of thicknesses of $\sim 2500$, 5000, 7500, and $10000 \AA$, respectively.

a field across the $a$-Se film. The magnitude of this field depended on the amount of dark current flowing through the film during charging. This dark current is known ${ }^{18}$ to originate due to transportation of electrons towards the positively charged top surface under the existing field. The dominant process for the availability of these electrons inside the $a$-Se film is known ${ }^{18}$ to be their injection from $\mathrm{Al}$ substrate into the $a$-Se film by surmounting the interfacial barrier height existing at the Al- $a$-Se interface. In this case, the barrier is due to the $\mathrm{P}(\mathrm{S}: \mathrm{AN})$ interface layer. During the above transportation of electrons a part of them get retrapped in photo-induced electron trap states existing at different energy levels in the films and part of them reach the top and get neutralized with the top positive surface charge. So this trapped charge (due to retrapping) appears as a net stored charge (residual potential) in $a$-Se films and is responsible for various peaks detectable in the TSD spectra. These charged samples were then dark rested for about $2 \mathrm{~h}$ for complete neutralization of the top surface charge and polarization to take place. In negatively charged $a$-Se films the polarization can also be achieved in a similar way as discussed above, the only difference, however, in this case being that the holes would be the trapped charge carriers.

Figure 2 shows TSD spectra of irradiated and positively charged $a$-Se films with and without the incorporation of $\mathrm{P}(\mathrm{S}: \mathrm{AN})$ interface layer. Curve A corresponds to only $a$-Se films, i.e., without interface layer, whereas curves B, C, D, and $\mathrm{E}$ correspond to $a$-Se films incorporated with interface layer of $\mathrm{P}(\mathrm{S}: \mathrm{AN})$ of thicknesses of $\sim 2500,5000,7500$, and $10000 \AA$, respectively. It is seen from these curves that each case shows two relaxation peaks positioned around 310 and $374 \mathrm{~K}$ and designated as peak I and peak II, respectively. The activation energy $(U)$ associated with these peaks has been calculated using the initial rise method. ${ }^{19}$ The values of $U$ associated with these peaks have been found ${ }^{11,12}$ to be $\sim 0.99$ and $1.60 \mathrm{eV}$, respectively. Furthermore, the mechanism of origin of these peaks in a positively charged case has been attributed ${ }^{11,12}$ to the trapping of charge carriers (electrons) at relatively shallow and deep trapping levels, respectively. It is also seen from Fig. 2 (curves B, C, D, and E) that the incorporation of $\mathrm{P}(\mathrm{S}: \mathrm{AN})$ interface barrier layer results in the reduction of charge storage capacity (area under the TSD spectra) in $a$-Se films. In fact, the reduction in charge storage increases with the increase in the thickness of interface layer. Downloaded 09 May 2007 to 202.141.140.34. Redistribution subje

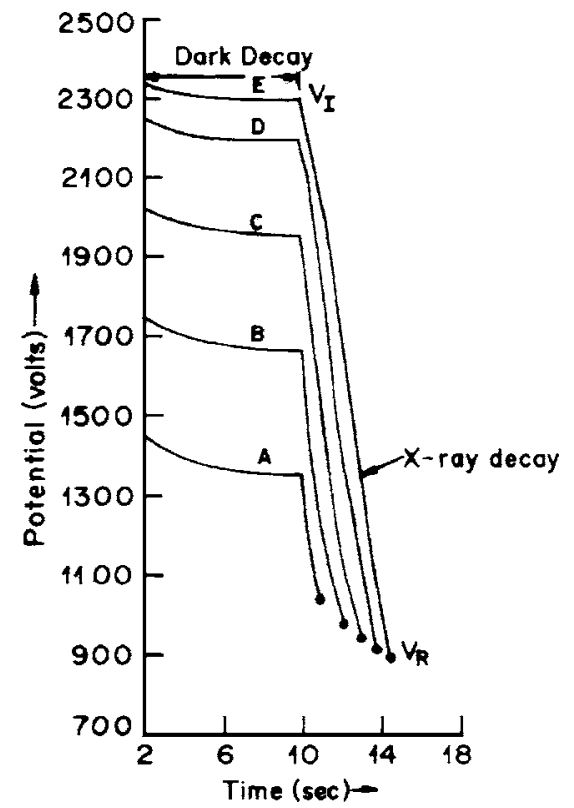

FIG. 3. Potential decay characteristics of $a$-Se films $(\sim 100 \mu \mathrm{m}$ thick $)$ with and without $\mathrm{P}(\mathrm{S}: \mathrm{AN})$ interface layer. Curve A corresponds to films without an interface layer and curves B, C, D, and E correspond to films with interface layers of $\mathrm{P}(\mathrm{S}: \mathrm{AN})$ of thicknesses $\sim 2500,5000,7500$, and $10000 \AA$, respectively.

Furthermore, it is seen that the effect of interface layer is more predominant on the relaxation peak I as compared to peak II. In fact, it becomes considerably reduced at interface layer of $10000 \AA$. This reduction in charge storage under the TSD spectra indicates indirectly that the built-up of residual potential in $a$-Se films decreases with the incorporation of $\mathrm{P}(\mathrm{S}: \mathrm{AN})$ interface barrier layer film. It may be mentioned here that the TSD spectra of $a$-Se films in negatively charged case have been found ${ }^{12}$ to be similar to the one in positively charged case except for the reversal in the polarity of the released current. Therefore, only one case, i.e., positive charging results, is presented in this letter.

To understand in a direct way the built-up of residual potential in $a$-Se films, the measurement of its surface potential as a function of $\mathrm{P}(\mathrm{S}: \mathrm{AN})$ barrier layer thicknesses has been studied using potential decay technique, as explained before. Figure 3 shows the decay of potential in dark and on $\mathrm{x}$-ray exposure of $a$-Se films with and without incorporation of $\mathrm{P}(\mathrm{S}: \mathrm{AN})$ interface layer. Curve A corresponds to pure $a$ -Se films, i.e., without interface layer, and curves B, C, D, and $\mathrm{E}$ correspond to $a$-Se films incorporated with interface layers of P(S:AN) of thicknesses of $\sim 2500,5000,7500$, and $10000 \AA$, respectively. It is from the curve that built-up in surface potential just after corona charging (acceptance potential in dark) increases from 1350 to $2300 \mathrm{~V}$ and the potential left after x-ray exposure (residual potential) decreases from 1040 to $890 \mathrm{~V}$ for a corresponding increase in $\mathrm{P}(\mathrm{S}: \mathrm{AN})$ layer of thickness from $\sim 0$ to $10000 \AA$. With this experimental data and Boag's ${ }^{20}$ expression for $S$ as given below in Eq. (1), we have calculated the values of $S$ both for pure as well as copolymer-incorporated $a$-Se films:

$$
S=\frac{1}{0.693 E}\left(\log V_{I}-\log V_{R}\right),
$$

where $V_{I}$ is the initial existing potential at the time of exposure, $V_{R}$ is the residual potential after x-ray exposure, and $E$ is the measured exposure rate and being $\sim 26 \times 10^{-3} \mathrm{R} / \mathrm{s}$, respectively. It may be mentioned here that though dark de-
to AlP license or copyright, see http://apl.aip.org/apl/copyright.jsp 
TABLE I. X-ray sensitivity parameters in $a$-Se films $(\sim 100 \mu \mathrm{m}$ thick $)$ incorporated with an interface layer of $\mathrm{P}(\mathrm{S}: \mathrm{AN})$ films.

\begin{tabular}{cccc}
\hline \hline $\begin{array}{c}\text { Thickness of P(S:AN) films } \\
(\AA)\end{array}$ & $\begin{array}{c}V_{I} \\
(\mathrm{~V})\end{array}$ & $\begin{array}{c}V_{R} \\
(\mathrm{~V})\end{array}$ & \multicolumn{1}{c}{$\begin{array}{c}S \\
\left(\mathrm{R}^{-1}\right)\end{array}$} \\
\hline 0 & 1350 & 1040 & 6.29 \\
2500 & 1670 & 980 & 12.85 \\
5000 & 1950 & 940 & 17.59 \\
7500 & 2190 & 910 & 21.17 \\
10000 & 2300 & 890 & 22.88 \\
\hline \hline
\end{tabular}

cay in each case of Fig. 3 remains practically constant yet to take into account the stabilized value of initial potential at the time of $\mathrm{x}$-ray exposure, the $\mathrm{x}$-ray exposure was made after $10 \mathrm{~s}$ in each case as shown in Fig. 3.

Putting the values of various parameters in Eq. (1), the values of $S$ for various cases mentioned above have been obtained and the electrical data so obtained have been given in Table I. It is seen from Table I that $S$ of $a$-Se films increases due to the incorporation of $\mathrm{P}(\mathrm{S}: \mathrm{AN})$ interface layer. In fact, it increases from $6.29 \mathrm{R}^{-1}$ in case pure $a$-Se films to $22.88 \mathrm{R}^{-1}$ for $a$-Se films incorporated with $\mathrm{P}(\mathrm{S}: \mathrm{AN})$ layer of $\sim 10000 \AA$.

The above-explained effects of $\mathrm{P}(\mathrm{S}: \mathrm{AN})$ interface layer on TSD behavior and surface potential decay (enhancement in $S$ ) of $a$-Se films may now be understood as follows. It is clear as explained above while discussing the polarization mechanism (Fig. 1) that the charge stored under the TSD peaks (Fig. 2) in $a$-Se films is primarily controlled by dark current, which in turn, is controlled by the charge carriers injected at the Al-a-Se interface. It is because if less is the number of charge carriers injected at the interface then less will be the dark current, higher will be the acceptance potential/field existing across the film, higher will be the mobility of the charge carriers, faster would be their transportation, less would be the probability of retrapping and recombination, and hence less will be the built-up of residual potential/charge stored in the $a$-Se film. The above discussion, therefore, shows that the barrier existing at the interface is very vital in governing the electrical properties of $a$-Se films, i.e., the higher is the barrier/blocking at the interface, less will be the injected carrier density and ultimately the less stored charge as explained above.

In fact, in the present case of $\mathrm{P}(\mathrm{S}: \mathrm{AN})-a$-Se combination, it is precisely this above-explained role of blocking which is played by $\mathrm{P}(\mathrm{S}: \mathrm{AN})$ interface film as shown in Fig. 1. However, the effective blocking of $\mathrm{P}(\mathrm{S}: \mathrm{AN})$ layer for injection of charge carriers from the $\mathrm{Al}$ substrate to the $a$ -Se films has been attributed to combined effect of two factors. Firstly, it provide a direct barrier for charge injection due to its insulating polymeric nature. Secondly, P(S:AN) layer may trap the injected charge carriers (which insulating polymer/copolymers normally do and show electret effect) that provides an internal space charge field, which resists further injection and transportation of the electrons across the interface barrier layer. Such an effective blocking would result in considerable reduction in charge storage capacity/ built-up of residual potential. Thus reduction in built-up of residual potential of $a$-Se films has been attributed to the above mentioned mechanism of blocking and charge trapping role of $\mathrm{P}(\mathrm{S}: \mathrm{AN})$ interface barrier layer. Besides the above-explained blocking role of interface layer, it also plays an important electret role on x-ray exposure. It means that Downloaded 09 May 2007 to 202.141.140.34. Redistribution subje the internal electret field (originating due to the trapping of the injected electrons) would enhance the transportation of holes that are produced on x-ray exposure and move towards the Al substrate and this result in achieving the low values of $V_{R}$ and hence enhanced $S$ in $a$-Se films. This indeed has been observed as shown by the charge stored under the two relaxation peaks (Fig. 2) and the values of $V_{I}$ and $V_{R}$ as shown in Fig. 3. Furthermore, as regards the predominant role of $\mathrm{P}(\mathrm{S}: \mathrm{AN})$ interfacial/blocking layer on peak I, it may now be easily understood that faster sweeping/enhanced mobility effects due to the copolymer film would be felt more at shallower traps (as compared to the relatively deep traps) to which this peak has been attributed and hence the probability of retrapping of charge carriers will be less at shallow traps as compared to the corresponding probability of their retrapping at relatively deeper levels. And this exactly has happened (Fig. 2) because there is relatively more reduction in the charge stored under peak I as compared to that of peak II due to the incorporation of $\mathrm{P}(\mathrm{S}: \mathrm{AN})$ interfacial/blocking layer. Thus it is clear from these investigations that $S$ of $a$-Se films increases with the incorporation of $\mathrm{P}(\mathrm{S}: \mathrm{AN})$ copolymer interface barrier layer. This is an important finding and may result in the development of new, better sensitive and cost effective imaging materials for xeroradiography (XR) photoreceptors, especially in a multilayer configuration incorporating the interface barrier layer of various suitable copolymeric materials.

In conclusion, it is stated that incorporation of an interfacial/blocking barrier layer of $\mathrm{P}(\mathrm{S}: \mathrm{AN})$ copolymer with $a$-Se films resulted in an overall reduction in its charge storage capacity, built-up of residual potential, and enhancement of its $S$. This has been attributed to the blocking and charge trapping effects of $\mathrm{P}(\mathrm{S}: \mathrm{AN})$ interface layer film.

The authors are thankful to Dr. Vikram Kumar, Director, National Physical Laboratory (NPL), Dr. A. K. Gupta, Head, Engineering Materials Division, and Dr. S. S. Bawa, In charge, Polymeric and Soft Materials Section, NPL for their keen interest and encouragement in this work.

${ }^{1}$ J. L. Donovan, J. Appl. Phys. 50, 6500 (1979).

${ }^{2}$ S. O. Kasap and S. Yannacopoulos, Can. J. Phys. 67, 686 (1989).

${ }^{3}$ J. Rowlands and S. Kasap, Phys. Today 50, 24 (1997).

${ }^{4}$ S. Yamada, A. Takahashi, H. Umazaki, M. Honda, K. Shiraishi, S. Rudin, C.-Y. Yang, and D. R. Bednarek, Proc. SPIE 3977, 429 (2000).

${ }^{5}$ S. Chand, J. Phys. D 31, 1049 (1998).

${ }^{6}$ M. Abkowitz and R. C. Enck, Phys. Rev. B 25, 2567 (1982).

${ }^{7}$ M. Abkowitz, F. Jansen, and R. R. Melnyk, Philos. Mag. B 51, 405 (1985).

${ }^{8}$ M. Abkowitz, in Disordered Semiconductors, edited by M. A. Kastner, G. A. Thomas, and S. R. Ovshinsky (Plenum, New York, 1987), p. 205.

${ }^{9}$ M. Abkowitz, Philos. Mag. Lett. 58, 53 (1988).

${ }^{10}$ S. O. Kasap and V. Aiyah, J. Appl. Phys. 69, 7078 (1987).

${ }^{11}$ M. N. Kamalasanan and S. Chand, Appl. Phys. Lett. 56, 330 (1990).

${ }^{12}$ S. Chand, R. C. Bhatheja, G. D. Sharma, and S. Chandra, J. Appl. Phys. 70, 5122 (1991)

${ }^{13}$ S. Chand, G. D. Sharma, R. C. Bhatheja, and S. Chandra, Appl. Phys. Lett. 59, 3514 (1991)

${ }^{14}$ S. Chand, G. D. Sharma, R. C. Bhatheja, and S. Chandra, Appl. Phys. Lett. 61, 1915 (1992)

${ }^{15}$ M. Kastner, D. Adler, and H. Fritzsche, Phys. Rev. Lett. 37, 1504 (1976).

${ }^{16}$ N. Mott, Contemp. Phys. 26, 203 (1977).

${ }^{17}$ S. R. Elliott, J. Non-Cryst. Solids 81, 71 (1986).

${ }^{18}$ R. M. Schaffert, in Electrophotography, edited by W. F. Berg (Focal, New York, 1971), pp. 45 and 265.

${ }^{19}$ C. F. J. Garlick and A. F. Gibson, Proc. Phys. Soc. London 60, 574 (1948).

${ }^{20}$ J. W. Boag, Phys. Med. Biol. 18, 3 (1973). 